\title{
The interpretations of scalar implicatures, presuppositions, and implicated presupposition by Thai children with autism
}

\author{
Nattanun Chanchaochai*
}

\begin{abstract}
Using the negated universal quantifier not every, the study investigates the interpretations of scalar implicatures, lexical presuppositions, and implicated presuppositions by Thai children with autism spectrum disorders (ASDs; $n=32$ ), compared to their typically-developing (TD) peers $(n=70)$ and adults $(n=40)$. The results provide further empirical evidence to the literature (Chevallier et al. 2010, Hochstein et al. 2017, Pijnacker et al. 2009) that not only do adolescents with ASD perform on par with TD adolescents, children with ASD are also age-appropriate in their performance on deriving scalar implicatures. Despite the children with ASD's ability to compute scalar implicatures, they still tend to give more logical, literal responses, compared to their peers. Compared to adults, both children with ASD and TD children still have a higher tendency to rely on the logical meaning rather than pragmatically inferred meaning. No additive effects of implicated presuppositions are found in any group of the participants.

Keywords. scalar implicatures; presuppositions; implicated presupposition; acquistion; typically-developing children; children with autism spectrum dirorder
\end{abstract}

1. Introduction. Autism Spectrum Disorder (ASD) is a developmental disorder defined by a dyad of impairments, including social communication impairments and restricted, repetitive patterns of behaviors and interests (American Psychiatric Association 2013). Pragmatic and discourse deficits have long been accepted to be central to the characteristics of ASD. Owing to the prevalence of pragmatic deficits across the spectrum, this domain has been the focal point of research for the past several decades (Kanner 1943, Tager-Flusberg 1999; a.o.). While the vast majority of literature on pragmatics and autism based its conclusions - that children with ASD have pragmatic deficits solely on the socially or contextually-dependent, less linguistically-informed side of pragmatics, first attempts on studying the linguistically-associated side found no difficulties for adolescents with ASD and adults on tasks involving scalar implicature (Chevallier et al. 2010, Hochstein et al. 2017, Pijnacker et al. 2009). These results suggest that certain less-explored parts of pragmatics in autism may still be intact.

The interplay between semantics and pragmatics plays a crucial role in the understanding of a language. Meaning in language is not always lexically encoded or grammatically derived. In addition to the literal, truth-conditional meaning, an utterance may also have other contextually influenced meanings. While the meaning inferred through particularized conversational implicatures (PCIs; Grice 1975, Levinson 1983, 2000) arises only by virtue of a particular conversation, not from any lexical or grammatical components of the utterance, the meaning implicated through

${ }^{*}$ This article is based on a part of my publicly defended $\mathrm{PhD}$ dissertation at the University of Pennsylvania. I would like to express my deepest gratitude to Florian Schwarz for his guidance and valuable advice. I would like to also thank David Embick, Kathryn Schuler, Jeremy Zehr, Muffy Siegel, other members of the Experimental Study of Meaning Lab at UPenn, and the audience at the first Experiments in Linguistic Meaning (ELM1) Conference. Author: Nattanun Chanchaochai, Chulalongkorn University (nattanun.c@chula.ac.th). 
generalized conversational implicatures (GCIs) is still linguistically tied. The latter type of pragmatic meaning, however, is still cancelable and not a part of the inherent, semantic meaning.

Presuppositions are another important type of inference in language, allowing more than one proposition to be communicated in one single sentence. They also serve as an indication of which proposition is the main assertion and which is merely background information (Sauerland 2008a). While the extent to which presuppositional inferences are semantically or pragmatically driven is still the subject of considerable debate, presuppositions are typically assumed to convey the information that is already known but taken for granted by the speakers and still be projected under embedding operators, unlike the literal content that can be canceled by these operators (Chierchia \& McConnell-Ginet 1990, Karttunen 1973; a.o.).

Additionally, Heim (1991) observed that the infelicities of certain expressions cannot be accounted for by either lexical presuppositions or conversational implicatures. She proposed the MAXIMIZE PRESUPPOSITION maxim, raising the possibility that implicated meanings could also be at play at the level of presuppositional information. Her maxim suggests that the form with the strongest lexical presupposition must be chosen whenever its presupposition is felicitous. In other words, an utterance should lexically presuppose as much as possible. The idea of implicated presupposition (Sauerland 2003, 2008a,b) is that in the case where the lexical entry with the strongest lexical presuppositions is not chosen, it can be implicated that the left-out presuppositions are not assumed. While it is claimed that implicated presuppositions are pragmatically derived in the same fashion as implicature, they are still backgrounded in the presuppositional domain. This hybrid type of inference between implicature and presupposition is thus interesting in its theoretical stance and in an acquisition point of view.

By exploring both the linguistically-informed and contextually-informed meaning of an utterance, this paper provides insights on what aspects of meaning in language are particularly difficult for children with ASD. The present paper is structured as follows. Section 2 provides the background on previous experimental studies on quantifiers. Section 3 then proceeds to present the methods of our experiment. The results are provided in Section 4 and discussed in Section 5.

2. Previous experimental studies. This section reviews the relevant literature on Semantic and pragmatic inferences of the quantifier 'every' (Section 2.1), previous experimental studies on quantifiers in child language (Section 2.2), and on quantifiers and autism (Section 2.3).

2.1. SemantiC AND PRAGMATIC INFERENCES OF THE QUANTIFIER 'EVERY'. Yatsushiro (2008) viewed that the oddness of the following utterances in (1) are due to the fact that they violate the three presuppositions of the universal quantifier 'every' in (2), related to the set of its first argument.

(1) a. \#Every tail of mine is long and curly.

b. \#Every tongue of mine is pink.

c. \#Every leg of mine is muscular.

(2) a. Existential presupposition: There exists at least one member.

b. ANTI-UNIQUENESS presupposition: There exists more than one member.

c. Anti-DUALITY presupposition: There exists more than two members.

While EXISTENTIAL presupposition is a part of the lexical meaning of the quantifier 'every', the other two are implicated presuppositions, whereby there is an expression with a stronger presup- 
position, e.g., 'the' for (2-b) and 'both' for (2-c), for a speaker to use. Speakers are required to choose the term that has the strongest presupposition, abiding by the pragmatic maxim of MAXIMIZE PRESUPPOSITION (Heim 1991). Hence, it is more felicitous to say the following sentences in (3) than the previous (1).

(3) a. My tongue/The tongue of mine is pink.

b. Both of my legs are muscular.

Quantifiers, such as 'every', may also involve scalar implicatures. Orderings on lexical scales express scalar relations between scalar alternatives within the same set, based on their semantic strength defined by entailment. A given lexical item outranks, i.e., is stronger than, its alternate on the same scale if and only if a statement with its presence unidirectionally entails the corresponding statement containing its alternate (Horn 1972). Scalar implicatures may hold in the case of numbers on numeric scales, where the situations that are compatible with an utterance described with a larger number are a superset of the situations that are compatible with an utterance with a smaller number. Similarly, assuming that some and all are scalar alternatives, the use of the expression some, which is ordered lower on the scale of quantity than the expression all, implicates that the use of all is not applicable, as seen that the utterance (4-a) typically implicates (4-b).

(4) a. Some graduate students have finished writing their term paper.

b. Not all graduate students have finished writing their term paper.

2.2. QuANTIFIERS IN CHILD LANGUAGE. Many studies have investigated children's comprehension of concepts related to quantification, including approximation, numbers, sets, and quantifiers (see Lidz (2016) and Smits (2010) for a comprehensive review). Noveck (2001) recruited 8-yearolds, 10-year-olds, and adult native speakers of French to participate in the study. Compared to the adults, the children in both groups were significantly more accepting of sentences such as 'Some giraffes have long necks', suggesting that children prefer more logical responses than adults. Some subsequent studies yielded similar results as Noveck (2001) (see Gualmini et al. 2001, Foppolo et al. 2012; a.o.), with one major note that the children's dispreference for calculating scalar implicatures may not arise from their genuine inability to do so but may be due to experimental settings. Papafragou \& Musolino (2003) found that 5-year-old Greek-speaking children were highly significantly more likely than adults to judge pragmatically infelicitous descriptions as being true. However, when they adjusted the experimental procedures and provided some training to another group of 5-year-olds, they observed a significantly higher rejection rates than those in the first version of experiment, although the children still did not reach the adult-like levels.

As laid out earlier, the universal quantifier 'every' may also involve inferences from lexical and implicated presuppositions. Yatsushiro (2008) reported that 6-year-old German-speaking children were more likely than adults to accept an equivalent sentence of 'every girl here is playing soccer' even when the picture they were shown depicted only one girl playing soccer. This suggests that children may base their felicity judgement merely on the lexical EXISTENTIAL presupposition. The results support that lexical presuppositions are acquired earlier than implicated presuppositions of ANTI-UNIQUENESS, which asks for more than one member in the set. Moreover, Yatsushiro (2008) found further, although less concrete, evidence for her hypothesis that the acquisition of implicated presuppositions and scalar implicatures may pattern together in their path. 
2.3. QUANTIFIERS AND AUTISM. First attempts at studying the linguistically-associated side found no difficulties for adolescents with ASD and adults on tasks involving scalar implicature. Pijnacker et al. (2009) found no statistical difference between high-functioning ASD and TD controls in their responses in judging underinformative some sentences such as 'Some sparrows are birds' and underinformative disjunction sentences such as 'Zebras have black or white stripes' to be false. Chevallier et al. (2010) followed up on Pijnacker et al. (2009), using spoken language stimuli with added stress on the disjunction $O R$. Contrary to their prediction, they replicated the unexpected results in Pijnacker et al. (2009) that adolescents with ASD equally accept pragmatically inferred disjunction as being correct, compared to the TD group. Hochstein et al. (2017) also reported similar results in adolescents with ASD $(n=18$; Age $M=14.9$, range $=12-18)$, compared to neurotypical adults $(n=17$; Age $M=22.6$; range $=18-41)$. However, they found that adolescents with ASD over-computed scalar implicatures in a different task where the participants need to base their answer on another person's epistemic state.

2.3.1. THE PRESENT STUDY. This study employs the negated universal quantifier not every to more directly compare between scalar implicature and the two types of presupposition within one paradigm. Similar to the universal quantifier every, the negated quantifier not every also yields an EXISTENTIAL presupposition and an ANTI-UNIQUENESS implicated presupposition. Additionally, the literal none meaning is also present. Given that if there is no intersection between restrictor and nuclear scope, cf. subject and predicate, other quantifiers, such as no or none, that are scalar alternatives to not every, would have been used to obey with the Maxim of Quantity, the meaning that there has to be a restrictor-nuclear scope intersection is then derived from the use of not every through scalar implicature. The four types of meanings for 'not every', derived from different mechanisms, are provided with Example (5) below.

(5) 'Not every boy is holding an ice cream.'

a. $\exists_{P s}$ (DOM): Existential presupposition for the domain

There is a boy.

b. $\quad \exists_{\text {Imp }}$ (RESTR $\cap$ SCOPE): Restrictor-nuclear scope intersection implicature There is a boy holding an ice cream.

c. $\quad>1$ IMPPS (DOM): Anti-uniqueness implicated presupposition

There is more than one boy.

d. $\neg \forall$ : Literal None meaning

It is not true that every boy is holding an ice cream.

3. Methods and design. This study adapted the Covered Box paradigm (Huang et al. 2013). In each trial, a context picture was first shown on a screen to the participants (see the top picture in Figure 1, with an auditory description in (6). The context picture depicts a group of animals doing the same thing, corresponding with the auditory context sentence. After the sentence ended, the screen was shifted to presenting two pictures, one visible and one covered with a black box, hidden from their view (see the bottom picture in Figure 1 for illustration). Note that in the visible pictures, the number of animals by type matched between the context screen and the test screen. Another description was then auditorily presented with the scheme provided in (7). The participants were instructed to choose either the visible picture or the covered box as matching with the auditory 
description. Prior to the critical trials, four practice trials were presented. In the practice trials, the black box was removed to reveal the picture behind the box. In the critical trials, on the other hand, no picture behind the covered box was revealed. The participants made their choice by selecting the picture that they thought was a good match with the sentence in (7).

naj klùm ní: sàt $\mathrm{t}^{\mathrm{h} u ́ k}$ tuia $y$ jù: $\quad \ldots$

in group this animal every CLS $y$ CONT ...

'In this group, every animal is doing $y . . . '$

... tè:-wâ: naj klùm ní: $x$ mâj thúk tu:a $y$ jù:

... but in group this $x$ not every CLS $y$ CONT

'but in this group, not every $x$ is doing $y$ '

[In this group, every animal is holding an ice cream, ...] (audio)

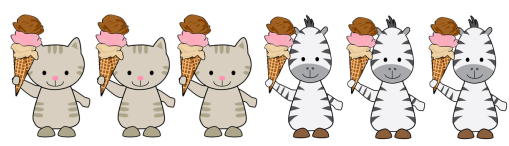

[...but in this group, not every zebra is holding an ice cream.] (audio)

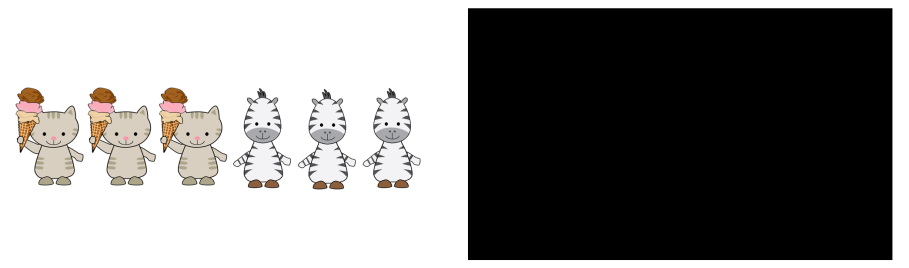

Figure 1: Example context screen (top) and test screen (bottom).

The conditions were manipulated with regards to the compatibility between the visible picture and the readings under investigation. To test how each of the four meanings, presented earlier in (5), plays a role in the participants' interpretation of the quantifier Not Every, four experimental conditions were created. All of the critical experimental conditions are consistent with the literal None $(\neg \forall)$ reading. They differ, however, in their consistency with the other three readings. Table 1 presents example visible pictures in the test screens for each experimental condition and summarizes predictions of compatibility with the interpretations under investigation for each condition. The study consisted of 64 critical trials (16 trials per condition). In addition, 48 filler trials with the quantifier some (3 conditions; 16 trials per condition; see Figure 2) were included to control for participants' understanding of the task. Those trials were counterbalanced and pseudo-randomized across 4 experimental lists. Each list, therefore, contained 16 critical trials and 12 filler trials, presented with an even distribution of trial types in each of the four blocks. Experimental lists were counterbalanced between participants.

3.0.1. Procedure And subgroups of Participants. A total of 92 children in the elementary grades were recruited from Kasetsart University Laboratory School, Center for Educational Research and Development. There were 32 children with ASD (3 Female; $M$ Age $=9 ; 8 ; M$ NVIQ $=95.5$ ) and 60 TD children (11 Female; $M$ Age $=7 ; 11 ; M$ NVIQ = 116.6). All the participants with ASD were classified in their medical records as having Autistic Disorder (AD). Children with 


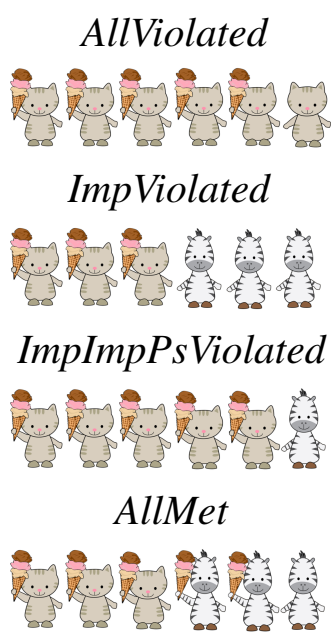

$$
\exists_{P s}(\text { Dom }) \quad \exists_{\text {Imp }}(\text { Restr } \cap \text { Scope }) \quad>1 \text { ImpPs (Dom) } \quad \neg \forall
$$

*

$\checkmark$

$\checkmark$

$\checkmark$
$*$

$*$

$*$

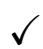

*

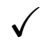

*

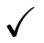

Note: $\checkmark$ indicates a visible picture selection; * indicated a covered box selection.

Table 1: Predictions for compatibility of readings in different conditions

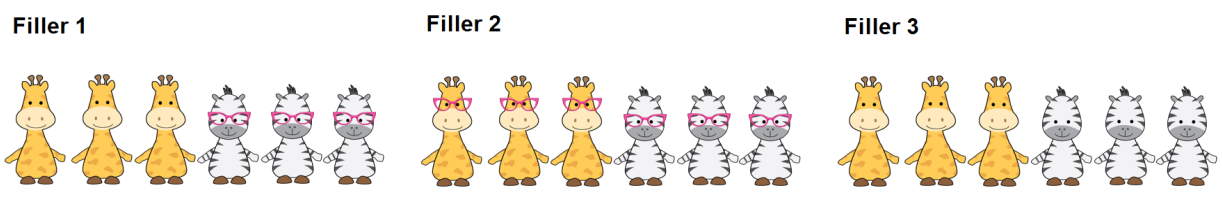

Figure 2: Filler conditions, shown with the auditory description “...only some animals...".

ASD were matched to their TD peers of the same gender in the same class. All of the participants had normal hearing and normal or corrected-to-normal vision. The studies were approved by the Institutional Review Board of the University of Pennsylvania. The parents of all the children provided written consent for them to participate in the study. The children and their parents were informed of their rights to withdraw from the study at any time. The children received toys and school supplies as compensation.

The child data were collected offline. An additional collection of adult data was done online using PennController (Zehr \& Schwarz 2018). The adult participants are native speakers of Thai, demographically mixed, recruited through personal contacts and word-of-mouth. They accessed the experiment online using their personal computer. The consent form was shown at the beginning of the experiment. Participants provided consent by their completion of the experiment. In the online version, participants were instructed to press $F$ on their keyboard to select the visible picture or $J$ to select the covered box. In the offline version, the participants indicated their choices by pointing. The only difference between the online and offline version of the task is that in the online version, before the experimental trials, there was a line of text on the screen emphasizing that their task is to match the sound with the second set of pictures on the screen in each trial.

To ensure objective measures on whether a participant was performing the task, only the participants whose accuracy score was over 50\% in the AllMet condition, the Filler 3 condition, or on 
average between AllMet and Filler 3 were included in the statistical analyses. The two conditions that the criteria are based on are not controversial in their interpretation with respect to their corresponding auditory sentences. These criteria were also used to subgroup children into Group 1 and Group 2 based on their performance.

Three adult participants did not meet the criteria and, therefore, were excluded from the statistical analysis. A higher proportion of children in each participant group was included in the performance group 2 for this task. Table 3.0.1 provides demographic details for each of the participant groups.

\begin{tabular}{l|ll|ll|ll}
\hline & \multicolumn{2}{|c|}{ Adults } & \multicolumn{2}{c|}{ ASD } & \multicolumn{2}{c}{ TD } \\
& Included & Excluded & Group 1 & Group 2 & Group 1 & Group 2 \\
\hline N (Female N) & $37(15)$ & $3(1)$ & $19(2)$ & $13(1)$ & $32(7)$ & $28(4)$ \\
\hline M Age & 32.43 & 37 & $10 ; 0$ & $9 ; 1$ & $8 ; 5$ & $7 ; 5$ \\
\hline M NVIQ & NA & NA & 99.79 & 89.24 & 120.71 & 111.97 \\
\hline
\end{tabular}

Table 2: Participant information

3.0.2. PREDICTIONS. This experiment aimed at exploring the acquisition of presuppositions, implicated presuppositions, and scalar implicature. The readings associated with 'not every' are laid out in (5) to gauge which reading is available for the participants' interpretation of the negated quantifier. These readings are associated with a predicted response pattern across the experimental conditions, summarized in Table 1 . The compatibility with each reading was assessed to create unique contrasts across conditions. The $\exists_{P s}$ (DOM) presupposition reading (5-a) predicts a contrast between the ImpImpPsViolated (choice of visible picture) and the AllViolated (covered) conditions. The $\exists_{I m p}$ (RESTR $\cap$ SCOPE) scalar implicature reading (5-b) predicts a contrast between the AllMet (visible) and the ImpViolated (covered) conditions. The >1 IMPPS (DOM) implicated presupposition reading (5-c) predicts a contrast between the ImpViolated (visible) and the ImpImpPsViolated (covered) conditions. It is worth noting, however, that the difference between the ImpImpPsViolated and the ImpViolated conditions would be due to an additive and not pure effects from implicated presuppositions alone, as scalar implicatures are also violated in the condition. Additionally, the pure literal $\neg \forall$ reading (5-d) predicts a contrast between different groups of participants if they accept the AllViolated visible picture to a different extent.

3.0.3. Modeling. Covered box rates were modelled separately for adults and children in the performance group 1. The child model contained 5 fixed effects factors, including condition (AllViolated, ImpViolated, ImpImpPsViolated, and AllMet), participant group, z-scored Ravens nonverbal IQ, z-scored age, and gender. The model additionally include interactions between condition and participant group. The adult model contained 3 fixed factors, including condition, $z-$ scored age, and gender and no interaction. Both models had a random effects factor for individual participants. Dummy coding was employed for baseline re-levelling.

Additionally, to compare the results between children and adults, one additional model was fitted to the covered box rates, using 3 fixed effects factors of condition, participant group, and gender. Interactions between condition and participant group were included, with a random effects factor for individual subjects. This model has to drop 2 fixed effects factors of age and NVIQ because no NVIQ data were collected for adults and age correlates with participant groups. 
Mixed effects logistic regression models were run using the lme4 package (Version 1.1.12; Bates et al. 2015) with the extension lmerTest package (Version 2.0.32; For obtaining p-values; Kuznetsova et al. 2016) in the R software (Version 3.3.1; R Core Team 2016) and MuMIn package (Version 1.43.6; For obtaining r-squared; Barton 2019).

4. Results. A mixed effects logistic regression model was fitted to the covered box responses of the adult participants. Covered responses were chosen significantly more in the AllViolated condition, compared to the other three conditions, including the ImpViolated condition $(\beta=-1.238$, $p=0.02)$, the ImpImpPsViolated condition $(\beta=-1.866, p<0.001)$, and the AllMet condition $(\beta=-$ 9.048, $p<0.001)$. At the same time, the participants chose the visible picture to a significantly higher extent in the AllMet condition than the other conditions: the ImpViolated condition ( $\beta=-$ $7.810, p<0.001)$ and the ImpImpPsViolated condition $(\beta=-7.182, p<0.001)$. The ImpViolated and the ImpImpPs Violated conditions turned out to not differ from each other in their covered box rates $(\beta=-0.628, p=0.119)$. Additionally, on average of all conditions, female participants were found to accept the visible picture to a significantly higher rate than men $(\beta=1.304, p=0.05)$.

According to the mixed effects logistic regression model on the Group 1 child data, children with ASD and the TD children in Group 1 displayed the same pattern of significance contrasts between conditions as adults. In particular, the children with ASD selected the covered box significantly more in the AllViolated condition, compared to the other three conditions, including the Imp Violated condition $(\beta=-1.731, p<0.001)$, the ImpImpPs Violated condition $(\beta=-2.411$, $p<0.001)$, and the AllMet condition $(\beta=-3.960, p<0.001)$. The visible picture was also chosen significantly more in the AllMet condition than the ImpViolated condition $(\beta=-2.228, p<0.001)$ and the ImpImpPsViolated condition $(\beta=-1.549, p=0.001)$. The ImpViolated and the ImpImpPsViolated conditions appeared to be similar in their covered box rates $(\beta=-0.679, p=0.082)$.

Similarly, the TD children exhibited significantly stronger preference for the covered box in the AllViolated condition than the ImpViolated condition $(\beta=-2.614, p<0.001)$, the ImpImpPsViolated condition $(\beta=-2.932, p<0.001)$, and the AllMet condition $(\beta=-5.041, p<0.001)$. The AllMet condition, on the other hand, significantly differed in its responses from the ImpViolated condition $(\beta=-2.428, p<0.001)$ and the ImpImpPsViolated condition $(\beta=-2.109, p=0.001)$. The ImpViolated and the ImpImpPsViolated conditions also did not differ in the TD group $(\beta=-0.319, p=0.290)$.

Additionally, group difference between the children with ASD and the TD children lies in their covered box rates in the AllViolated condition, with the children with ASD choosing significantly fewer covered responses $(\beta=1.561, p=0.02)$. Moreover, on average for all the children in the performance group 1, age ( $\beta=0.682, p<0.01)$, but not NVIQ ( $\beta=0.076, p=0.59)$, significantly increases their covered responses. Figure 3 and 4 plotted mean covered response rates in adults and children, respectively. Significance levels from the two models presented above are also summarized in the two figures.

To further compare between the adult and child data, a logistic mixed effects model was fitted to their data, dropping the fixed effects of NVIQ and age. In general, adults were significantly more likely than both groups of children to choose a covered box in all of the conditions, except in the AllMet condition, where children chose a covered box significantly more than adults. Comparing the differences between (1) the AllViolated and the ImpImpPs Violated conditions (compared to ASD: $\beta=-0.59, p=0.374$; to TD: $\beta=-1.123, p=0.08$ ) and (2) the ImpViolated and the ImpImpPsVi- 


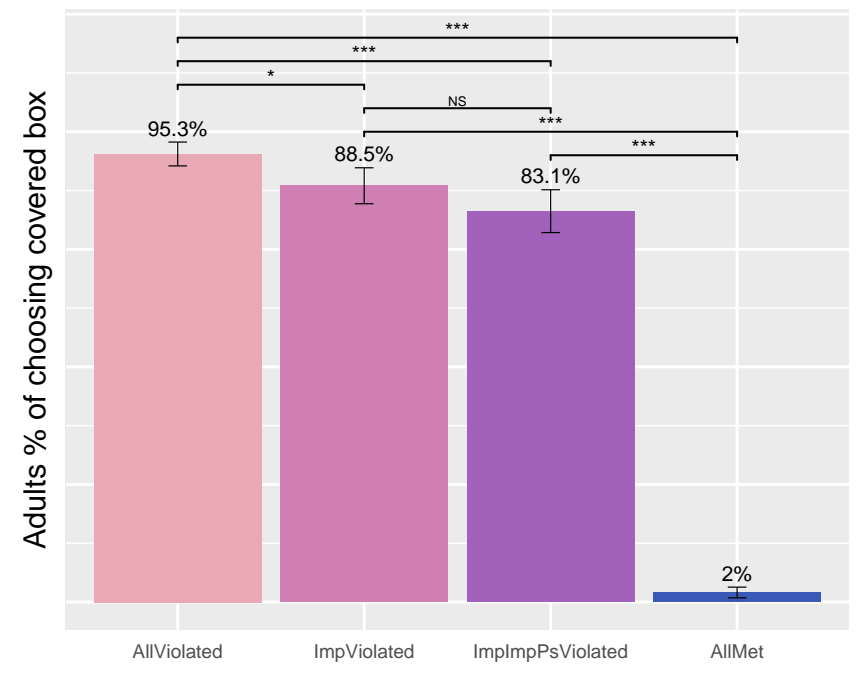

Figure 3: Adults' accuracy by condition
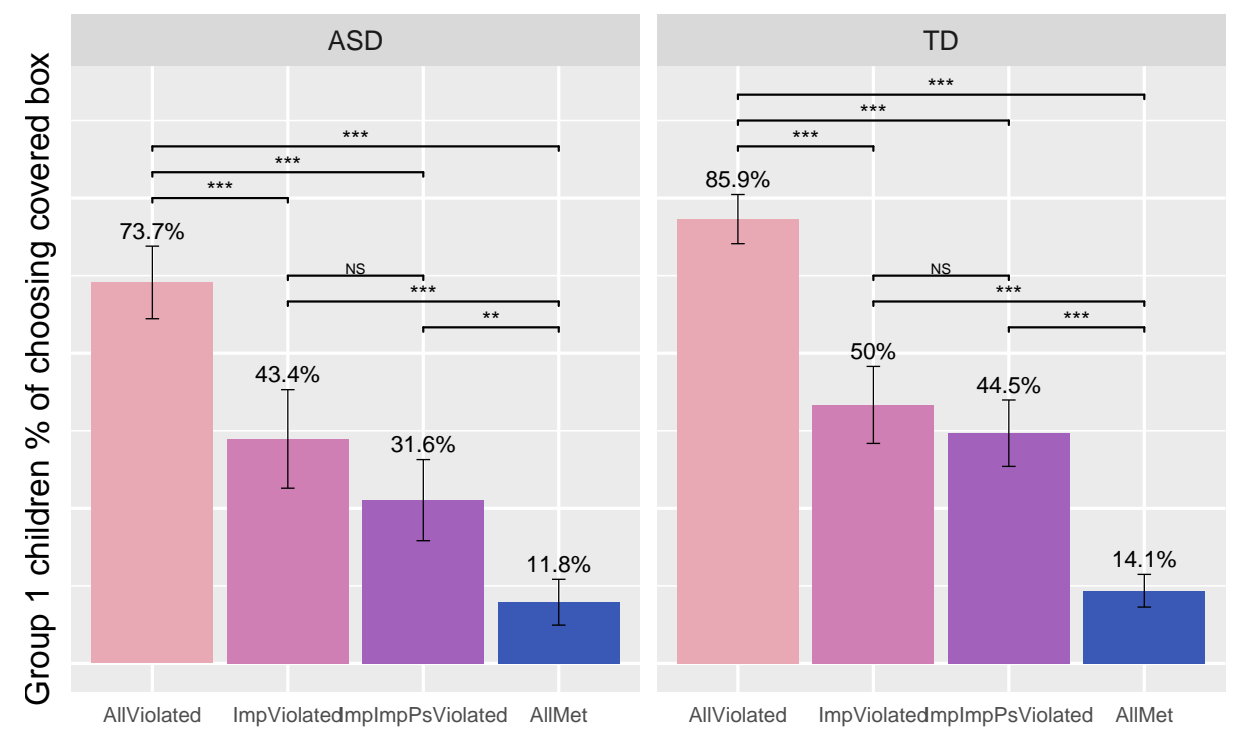

Figure 4: Group 1 children's accuracy by condition

olated conditions (compared to ASD: $\beta=-0.073, p=0.90$; to TD: $\beta=0.298, p=0.55$ ), adults did not differ from either group of children. However, adults differ from both groups of children in their differences between the covered box rates in the ImpViolated and the AllMet conditions (compared to ASD: $\beta=5.428, p<0.001$; to TD: $\beta=5.269, p<0.001$ ).

No logistic regression model was run on the data of the children in the performance group 2. In general, the children in this group seemed to misunderstand or not fully comprehend the task, resulting in similar pattern of covered box rates across conditions (ASD: 65.4\% for AllViolated, $75 \%$ for ImpViolated, 71.2\% for ImpImpPsViolated, and 63.5\% for AllMet; TD: 89.3\% for AllViolated, $81.2 \%$ for ImpViolated, $82.1 \%$ for ImpImpPsViolated, and $90.2 \%$ for AllMet). 
5. Discussion. The adult data provide empirical evidence that they were accessing the meanings from the EXISTENTIAL lexical presupposition and the restrictor-nuclear scope intersection implicature, as suggested from their significant differences in covered box rates between (1) the AllViolated and the ImpImpPsViolated conditions and (2) the ImpViolated and the AllMet conditions, respectively. The adults, however, did not display their sensitivity to the ANTI-UNIQUENESS implicated presuppositions, showing no differences between the ImpImpPsViolated and the ImpViolated conditions. The pattern in the child data was the same as adults, with signs of them computing lexical presuppositions and scalar implicatures, but not implicated presuppositions. Overall, age was a significant factor in predicting their responses, with older children choosing more covered boxes. While the overall patterns are similar, the children with ASD chose covered boxes significantly less in the Allviolated condition, compared to the TD children. Other pairs of conditions did not yield such differences. This suggests that access of the literal, logical meaning is the most indicative of group differences in children, with children with ASD basing their interpretation on literal meanings to a higher extent than TD children. In contrast, children with ASD are on par with TD children in accessing the meaning derived from lexical presuppositions, implicated presuppositions, and scalar implicatures.

A comparison between adults' and children's behavior reveals several significant differences. For one thing, adults had significantly higher covered box rates in the AllViolated, the ImpViolated, and the ImpImpPsViolated conditions, while having significantly lower covered box rates in the AllMet condition than both groups of children, suggesting that they were, in general, more likely to produce expected results. Secondly, adults were significantly less likely to accept the visible picture in the Allviolated condition than both children with ASD and TD children, indicating the children's higher tendency to rely on the logical meaning rather than pragmatically inferred meaning, compared to adults. Additionally, adults significantly chose more covered boxes in the ImpViolated condition than in the AllMet condition to a higher extent than children in both groups. This suggests that children are less likely to derive scalar implicatures, compared to adults. The adults and both groups of the children, however, are not different in their likelihood to derive lexical presuppositions. The additive effect of implicated presupposition violation is also not greater in the adults, compared to the groups of children.

Overall, the results are very consistent with previous literature in many aspects. Firstly, previous experimental studies on scalar implicature in child language found significantly higher preference for logical responses to scalar implicature in children than adults (Gualmini et al. 2001, Foppolo et al. 2012, Noveck 2001, Papafragou \& Musolino 2003; a.o.). The current study also observed that children rely more on logical, literal meaning to a higher extent compared to adults.

Secondly, studies on scalar implicatures in adolescents with ASD suggest that their scalar implicatures are intact (Chevallier et al. 2010, Hochstein et al. 2017, Pijnacker et al. 2009). The results of this study provide further empirical evidence to the literature that not only do adolescents with ASD perform on par with TD adolescents, children with ASD are also age-appropriate in their performance on deriving scalar implicatures. Additionally, this study adds that even though the children with ASD's ability to compute scalar implicature is on par with TD children, they still tend to give more logical, literal responses, compared to their peers, as seen in their significantly lower covered box rates in the Allviolated condition, and not in other conditions. 
Thirdly, the current results are in accordance with Yatsushiro (2008)'s claim that lexical presuppositions are acquired earlier than scalar implicatures and perhaps than certain types of implicated presuppositions, similar to Legendre et al. (2011). The current results also adds to the literature that types of implicated presupposition matter in the acquisition pattern. This is evident from the fact that even though implicated presuppositions seem to affect the accuracy rates in comprehending certain personal reference terms (Chanchaochai 2017), they do not seem to have an additive effect in this study of the negated quantifier. The proposal that different types of implicated presuppositions may affect participants' performance differently is a plausible proposal, considering that rates of deriving scalar implicatures were also previously observed to differ by scalar terms. Papafragou \& Musolino (2003) improved success rates in children deriving scalar implicatures when the task involved number terms, such as $<$ three, two $>$, rather than scalar terms, such as $<$ all, some $>$. These observations raise interesting theoretical issues on types of implicated presupposition and their pattern of acquisition. Further investigation into the acquisition of different types implicated presuppositions, compared to other types of pragmatic inferences, should be made, probing the pure, not additive, effects from implicated presuppositions.

\section{References}

American Psychiatric Association. 2013. Diagnostic and statistical manual of mental disorders (5th ed.). Arlington, VA: American Psychiatric Association.

Barton, Kamil. 2019. Mumin: Multi-model inference. https://CRAN.R-project.org/ package=MuMIn. R package version 1.43.6.

Bates, Douglas, Martin Mächler, Ben Bolker \& Steve Walker. 2015. Fitting linear mixed-effects models using lme4. Journal of Statistical Software 67(1). 1-48. 10.18637/jss.v067.i01.

Chanchaochai, Nattanun. 2017. On acquiring a complex personal reference system: Experimental results from thai children with autism. Poster presented at Sinn und Bedeutung 22, September 7-10.

Chevallier, Coralie, Deirdre Wilson, Francesca Happé \& Ira Noveck. 2010. Scalar inferences in autism spectrum disorder. Journal of Autism and Developmental Disorders 40. 1104-1117.

Chierchia, Gennaro \& Sally McConnell-Ginet (eds.). 1990. Meaning and grammar. an introduction to semantics. Cambridge: MIT Press.

Foppolo, Francesca, Maria Teresa Guasti \& Gennaro Chierchia. 2012. Scalar implicatures in child language: Give children a chance. Language Learning and Development 8. 365-394.

Grice, Herbert Paul. 1975. Logic and conversation. In Peter Cole \& Jerry L. Morgan (eds.), Syntax and semantics: Speech acts, vol. 3, 41-58. New York: Academic Press.

Gualmini, Andrea, Stephen Crain, Luisa Meroni, Gennaro Chierchia \& Maria Teresa Guasti. 2001. At the semantics/pragmatics interface in child language. In Rachel Hastings, Brendan Jacksonand \& Zsofia Zvolenszkyn (eds.), Proceedings of the 11th Semantics and Linguistic Theory (SALT11) Conference, 231-247. Ithaca, NY: CLC-Publications, Cornell University.

Heim, Irene. 1991. Artikel und Definitheit. In Von A. Stechow \& D. Wunderlich (eds.), Semantik: Ein internationales Handbuch der zeitgenoessischen Forschung, 487-535. Berlin: De Gruyter.

Hochstein, Lara, Alan Bale \& David Barner. 2017. Scalar implicature in absence of epistemic reasoning? The case of autism spectrum disorder. Language Learning and Development. 
Horn, Laurence R. 1972. On the semantic properties of logical operators in English: Department of Linguistics, University of California, Los Angeles Doctoral dissertation.

Huang, Yi Ting, Elizabeth Spelke \& Jesse Snedeker. 2013. What exactly do numbers mean? Language Learning and Development 9. 105-129.

Kanner, Leo. 1943. Autistic disturbances of affective contact. Nervous Child 2. 217-250.

Karttunen, Lauri. 1973. Presuppositions of compound sentences. Linguistic Inquiry 4. 169-193.

Kuznetsova, Alexandra, Per Bruun Brockhoff \& Rune Haubo Bojesen Christensen. 2016. lmertest: Tests in linear mixed effects models. https://CRAN.R-project.org/package= lmerTest. R package version 2.0-32.

Legendre, Géraldine, Isabelle Barrière, Louise Goyet \& Thierry Nazzi. 2011. Quantifier acquisition: presuppositions of 'every'. In Mihaela Pirvulescu et al. (eds.), Selected Proceedings of the 4th Conference on Generative Approaches to Language Acquisition North America (GALANA 2010), 150-162. Somerville, MA: Cascadilla Proceedings Project.

Levinson, Stephen C. 1983. Pragmatics. Cambridge: Cambridge University Press.

Levinson, Stephen C. (ed.). 2000. Presumptive meanings: The theory of generalized conversational implicature. Cambridge: MIT Press.

Lidz, Jeffrey L. 2016. Quantification in child language. In Jeffrey L. Lidz, William Snyder \& Joe Pater (eds.), The oxford handbook of developmental linguistics, 498-519. Oxford: Oxford University Press.

Noveck, Ira A. 2001. When children are more logical than adults: experimental investigations of scalar implicature. Cognition 78. 165-188.

Papafragou, Anna \& Julien Musolino. 2003. Scalar implicatures: experiments at the semanticspragmatics interface. Cognition 86. 253-282.

Pijnacker, Judith, Peter Hagoort, Jan Buitelaar, Jan-Pieter Teunisse \& Bart Geurts. 2009. Pragmatic inferences in high-functioning adults with autism and asperger syndrome. Journal of Autism and Developmental Disorders 39. 607-618.

R Core Team. 2016. R: A language and environment for statistical computing. R Foundation for Statistical Computing Vienna, Austria. https: / / www.R-project.org/.

Sauerland, Uli. 2003. A new semantics for number. In Proceedings of SALT13, 258-275.

Sauerland, Uli. 2008a. Implicated presuppositions. In Anita Steube (ed.), Sentence and context, Mouton de Gruyter.

Sauerland, Uli. 2008b. On the semantic markedness of phi-features. In Daniel Harbour, David Adger \& Susana Bejar (eds.), Phi Theory: Phi Features across Interfaces and Modules, 5782. Oxford University Press.

Smits, Erik-Jan. 2010. Acquiring quantification: how children use semantics and pragmatics to constrain meaning: Department of Linguistics, University of Groningen Doctoral dissertation.

Tager-Flusberg, Helen. 1999. A psychological approach to understanding the social and language impairments in autism. International Review of Psychiatry 11. 325-334.

Yatsushiro, Kazuko. 2008. Quantifier acquisition: presuppositions of 'every'. In Proceedings of Sinn und Bedeutung 12, 663-677. Oslo: University of Oslo.

Zehr, Jérémy \& Florian Schwarz. 2018. Penncontroller for internet based experiments (IBEX) 10.17605/OSF.IO. https://doi .org/10.17605/OSF . IO/MD 832. 